\title{
Identification and genetic analysis of qCL1.2, a novel allele of the "green revolution" gene SD1 from wild rice (Oryza rufipogon) that enhances plant height
}

Lizhen Zhang ${ }^{1,2 \dagger}$, Jingfen Huang ${ }^{2,3 \dagger}$, Yanyan Wang ${ }^{2 \dagger}$, Rui Xu², Ziyi Yang ${ }^{1}$, Zhigang Zhao ${ }^{4}$, Shijia Liu ${ }^{4}$, Yunlu Tian ${ }^{4}$, Xiaoming Zheng ${ }^{2}$, Fei Li ${ }^{2}$, Junrui Wang ${ }^{2}$, Yue Song ${ }^{2}$, Jiaqi Li' ${ }^{2}$, Yongxia Cui ${ }^{2}$, Li-Fang Zhang ${ }^{1}$, Yunlian Cheng ${ }^{2}$, Jinhao Lan ${ }^{1^{*}}$, Weihua Qiao ${ }^{2^{*}}$ (D) and Qingwen Yang ${ }^{2^{*}}$

\begin{abstract}
Background: The exploitation of novel alleles from wild rice that were lost during rice cultivation could be very important for rice breeding and evolutionary studies. Plant height $(\mathrm{PH})$ was a target of artificial selection during rice domestication and is still a target of modern breeding. The "green revolution" gene semi-dwarf 1 (SD1) were well documented and used in the past decades, allele from wild rice could provide new insights into the functions and evolution of this gene.

Results: We identified a PH-related quantitative trait locus, $q C L 1.2$,from wild riceusing a set of chromosome segment substitution lines. aCL1.2encodesa novel allele of SD1 gene. The wild allele of SD1 is a dominant locus that can significantly promote rice internode length by regulating the expression levels of genes involved in gibberellin biosynthesis and signal transduction. Nucleotide diversity and haplotype network analyses of the SD1 gene were performed using 2822 rice landraces. Two previously reported functional nucleotide polymorphisms clearly differentiated japonica and indica rice; however, they were not associated with $\mathrm{PH}$ selection. Other new functional nucleotide polymorphisms in the coding, but not promoter, regions were involved in $\mathrm{PH}$ selection during rice domestication. Our study increasesunderstanding of the rice SD1 gene and provides additional evidence of this gene's selection during rice domestication.
\end{abstract}

Conclusions: Our findings provide evidence thatSD1 gene from wild rice enhances plant height and new functional nucleotide polymorphisms of this gene were artificially selected during cultivated rice differentiation.

Keywords: Wild rice, Chromosome segment substitution line, SD1, Plant height, Rice domestication

\footnotetext{
*Correspondence: jinhao2005@163.com; qiaoweihua@caas.cn;

yangqingwen@caas.cn

'Lizhen Zhang, Jingfen Huang and Yanyan Wang contributed equally to this

work.

'Qingdao Agricultural University, Qingdao 266109, China

${ }^{2}$ Institute of Crop Science, Chinese Academy of Agricultural Sciences, Beijing 100081, China

Full list of author information is available at the end of the article
}

(c) The Author(s). 2020 Open Access This article is licensed under a Creative Commons Attribution 4.0 International License, which permits use, sharing, adaptation, distribution and reproduction in any medium or format, as long as you give appropriate credit to the original author(s) and the source, provide a link to the Creative Commons licence, and indicate if changes were made. The images or other third party material in this article are included in the article's Creative Commons licence, unless indicated otherwise in a credit line to the material. If material is not included in the article's Creative Commons licence and your intended use is not permitted by statutory regulation or exceeds the permitted use, you will need to obtain permission directly from the copyright holder. To view a copy of this licence, visit http://creativecommons.org/licenses/by/4.0/ The Creative Commons Public Domain Dedication waiver (http://creativecommons.org/publicdomain/zero/1.0/) applies to the data made available in this article, unless otherwise stated in a credit line to the data. 


\section{Background}

Asian rice (OryzasativaL.) is a cultivated, inbred species that provides $35-60 \%$ ofdietarycaloriesto $\sim 50 \%$ of the world's population [1].Plant-architectureis crucial to the high yield of rice, and the ideal plant-architectureis essential for a high rice yield [2,3]. Plant height $(\mathrm{PH})$ is a main factor affecting rice plant-architecture, and an ideal $\mathrm{PH}$ is necessary for a high crop yield. $\mathrm{PH}$ is mostly determined by cell division, cell differentiation and cell expansion in the stem. Plant hormones, such as gibberellin (GA), play important roles in $\mathrm{PH}$ regulation [4]. In the 1960s, a mutation in the Taiwanese indica landrace 'Dee Gee Woo Gen' [5] led to a semi-dwarf variety of rice, known as IR8, which made an outstanding contribution to world food security, known as the rice "green revolution" $[6,7]$. The short PH of IR8 results from a mutation in the plant's semi-dwarf 1 ( $s d 1)$ gene, which islocated on the long arm of chromosome (Chr.) 1 and encodes an oxidase enzyme involved in GA biosynthesis [8]. A recessive allele, $s d 1$, caused by a 383-bp deletion in $S D 1$, is primarily responsible for the reduction in $\mathrm{PH}$ observed in most semi-dwarfs $[5,8$, 9]. At present, at least five different alleles, including the wild-type allele, $s d 1-d$ in 'Dee Gee Woo Gen,'sd1- $r$ in 'Reimei,' $s d 1-c$ in 'Calrose76' and $s d 1-j$ in 'Jikkoku' have been discovered $[5,9]$. Mutants of these alleles lead to different degrees of dwarfing through changes in $\mathrm{PH}$. However, the origin of the rice $s d 1$ allele and the role of $s d 1$ in rice domestication are still unclear.

Common wild rice (Oryza rufipogon Griff.), which has an AA genome similar to that of cultivated rice, is considered the ancestor of cultivated rice [10-12]. Wild rice has a greater genetic diversity than cultivated rice because genetic diversity was profoundly reduced during rice domestication [13]. Many novel alleles of genes controlling important agronomic traits in rice have been found in wild rice and its relatives, and they have provided an increased understanding of gene functions and the domestication process [14]. The development of chromosome segment substitution lines (CSSLs) through interspecific hybridization is a powerful platform for QTL mapping and gene cloning and produces useful genetic resource for genome research [15]. In our previous study, a set of CSSLs was constructed with wild rice as the donor parent and the indica cultivar 9311 as the recurrent parent. Many quantitative trait loci (QTLs) correlated with important agronomic traits have been identified using the CSSL platform [16-18]. In this study, we fine mapped a novel allele of the "green revolution" gene $s d 1$ using a CSSL population. The genetic analysis revealed that this allele from wild rice is a dominant locus that can significantly increase rice culm length. A previous report [19] suggested that $s d 1$ was subjected to artificial selection during rice evolution, and two single nucleotide polymorphisms (SNPs) of $s d 1$ can clearly differentiate the japonica landraces and wild rice. Nucleotide diversity and haplotype network analyses of the $s d 1$ geneconfirmed this hypothesis. However, these two SNPs were not associated with the $\mathrm{PH}$ phenotype. We found nine other functional nucleotide polymorphisms (FNPs) that were used in rice domestication owing to their influence on PH. Our study presents new evidence for artificial $\mathrm{PH}$ selection during rice domestication and differentiation, and the novel SD1 allele and the FNPs provide an increased understanding of rice $\mathrm{PH}$-targeted breeding.

\section{Results}

\section{$q \mathrm{CL} 1.2$ detection using a CSSL population}

In previously study, a set of CSSLs was constructed in our laboratory [10]. The donor wild rice parental plant has a procumbent phenotype. To identify genes controlling PH during rice domestication, we conducted a QTL analysis for $\mathrm{PH}$ using this CSSLs population. The PHs of the CSSLs were investigated under five environmental conditions (Table 1). The $\mathrm{PH}$ phenotype substantially differed within the CSSL population (Fig. S1). Genotyping was performed using 157 molecular markers, including 97 SSR markers and 60 InDel markers. The linkage map of SSR/InDel markers was shown in Fig. S2a. In total, 11 QTLs correlated with $\mathrm{PH}$ were identified under the five environmental conditions (Table 2). One QTL, located near InDel 1-16 on Chr. 1 was detected in four environments, had the highest LOD value (45.01 in E3) and explained $48 \%$ of the $\mathrm{PH}$ variance (Table 2), indicating that this QTLis likely a main effect QTL. This QTL was named as $q C L 1.2$. One CSSL, CSSL28, which had the greatest $\mathrm{PH}$ in the CSSL population and harboredqCL1.2, was selected for further study. The CSSL28 genotype is shown in Fig. S2a. Only two substituted segments from wild rice were detected using SSR/InDel markers in the

Table 1 The locations of rice crops used in this experiment

\begin{tabular}{|c|c|c|}
\hline Environment & Crop location & Cropping season \\
\hline E1 & 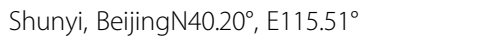 & Apr-Oct. 2017 \\
\hline E2 & Nanjing, Jingsu Province,N32.03ํ, E118.47º & May-Oct. 2017 \\
\hline E3 & Sanya, Hannan Province,N18.15, E109.31º & Dec.2017 -May 2018 \\
\hline E4 & 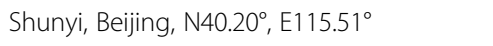 & April-Oct. 2018 \\
\hline E5 & Nanjing, Jingsu Province, N32.03, E118.47º & April-Oct. 2018 \\
\hline
\end{tabular}


Table 2 QTLs correlated with plant height in five environments identified using SSR/InDel genotypes detected in a CSSL population

\begin{tabular}{llllll}
\hline Marker & Chr. & Environment & LOD & PVE(\%) & Add \\
\hline InDe11-16 & 1 & E3 & 45.01 & 48.01 & 40.76 \\
& & E1 & 40.12 & 44.02 & 48.66 \\
& & E2 & 31.86 & 40.94 & 41.38 \\
& & E5 & 3.263 & 4.830 & 12.95 \\
RM125 & 7 & E3 & 14.85 & 11.04 & 22.76 \\
& & E5 & 13.67 & 10.97 & 28.28 \\
RM427 & 7 & E3 & 8.480 & 5.869 & -20.83 \\
& & E5 & 8.105 & 6.106 & -26.49 \\
RM5427 & 6 & E5 & 4.071 & 8.665 & 0.6553 \\
InDel4-3 & 4 & E1 & 6.979 & 6.726 & -22.43 \\
InDe16-4 & 6 & E2 & 6.886 & 10.60 & -62.14 \\
InDe11-12 & 1 & E2 & 5.409 & 8.197 & -38.72 \\
RM190 & 6 & E1 & 4.635 & 4.353 & -21.99 \\
RM128 & 1 & E4 & 4.438 & 9.150 & 32.54 \\
RM273 & 4 & E1 & 4.265 & 3.988 & 24.25 \\
RM533 & 7 & E2 & 2.774 & 4.084 & 17.41 \\
\hline PVE, the & & of &
\end{tabular}

PVE, the percentage of phenotypic variation explained; Add, the additive effect of the QTL

whole CSSL28 genome (Fig. S2a); therefore, CSSL28 was considered a near isogenic line (NIL) of $q$ CL1.2.

\section{Phenotypic characteristics of parental lines CSSL28 and 9311 and their $F_{1}$ generation}

CSSL28 showed a significantly greater $\mathrm{PH}$ than the recurrent parent 9311. The PH of CSSL28 was $180.3 \mathrm{~cm}$, while that of 9311 was $116.8 \mathrm{~cm}$ (in E5). The $\mathrm{F}_{1}$ was generated from a cross with CSSL28 as the female parent and 9311 as the male parent. The resulting $\mathrm{F}_{1}$ individuals were as tall as CSSL28 (Fig. 1a, b). A difference in $\mathrm{PH}$ between CSSL28 and 9311 was clear evident at the seedling stage (Fig. S2b), The difference was significant from30 d after sowing. The difference in $\mathrm{PH}$ between CSSL28 and 9311 was extremely significant at the heading stage, reaching $\sim 63 \mathrm{~cm}$ on average (Fig. 1c). The lengths of panicle and internodes of 9311 and CSSL28 were also measured (Fig. 1d, e). The basal three internodes of CSSL28 were similar in length to those of 9311. However, the upper three internodes and panicles of CSSL28 were longer than those of 9311. The second and third internodes of CSSL28 were longer than those of 9311 by $\sim 18.4$ and $\sim 17.7 \mathrm{~cm}$, respectively. The total lengths of second and third internodes in CSSL28 contributed approximately $43.8 \%$ to the total culm, as compared with $37.6 \%$ in 9311 (Fig. 1f). The increase in CSSL28 PH was mainly caused by elongated upper second and third internodes.
To determine the cause of the differences in $\mathrm{PH}$, histological observations of transverse and longitudinal sections of the internodes of CSSL28 and 9311 were recorded (Fig. 2). The transverse sections of the third internodes from the main culms indicated that the CSSL28 cells, especially the vascular cells, were much bigger than those of 9311. The longitudinal sections of the internodes suggested that there was no significant difference in cell length between CSSL28 and 9311. Similar results were observed for the second, fourth and fifth internodes. However, for the first and basal internodes, no differences between CSSL28 and 9311 were observed in the transverse sections. Because the stems of CSSL28 are much thicker than those of 9311, we deduced that the increased PH of CSSL28 resulted from an enhanced cell number and cell size at the first through fifth internodes, rather than an enhanced cell length.

\section{Fine mapping of $q C L 1.2$ and gene prediction}

The $\mathrm{F}_{2}$ population of CSSL28/9311, containing 402 individuals, was constructed for a genetic analysis in the summer of 2017. Thesegregation ratio of $\mathrm{PH}$ fit a 3:1 ratio $\left(X^{2}=1.76<X^{2} 0.05,1=3.84\right)$ for single gene inheritance. In2018, two segregating $\mathrm{F}_{3}$ populations derived from a single heterozygous plant were used for further genetic analyses. One $\mathrm{F}_{3}$ population, containing 1611 individuals, was planted in E3, and another one, containing 928 individuals, was plant in E5. As shown in Fig. 3a, b, the $\mathrm{PH}$ showed a bimodal distributionand similar 3:1 segregation ratios were obtained $\left(x^{2}=2.20<\chi^{2} 0.05,1=\right.$ $\left.3.84, \chi^{2}=3.31<\chi^{2} 0.05,1=3.84\right)$. These results indicated that the difference in PH between CSSL28 and 9311 was controlled by a single QTL, $q C L 1.2$.

We located $q C L 1.2$ between RM128 and RM472 (near InDel 1-16) on Chr. 1. To narrow the site of $q C L 1.2$ into a smaller region, we selected molecular markerswithin this interval. One InDel and eight SSR markers (InDel114、RM486、RM5389、RM11908、RM11986、RM11928、RM11960、RM11974、RM11982) with polymorphisms between CSSL28 and 9311 were seleced. Using $\sim 2000 \mathrm{~F}_{3}$ segregating individuals, qCL1.2 was narrowed to a 131-kb interval between RM11974 and RM11982 (Fig. 3c). According to the Rice Genome Annotation Rice Genome Annotation Project Database (http://rice.plantbiology.msu.edu/), this interval may include 13 candidate genes (Table S1), including the "green revolution" gene $s d 1$ (LOC_Os01g66100). By sequencing LOC_Os01g66100,wefound that the first and third exons in the coding region produced synonymous and non-synonymous SNP changes, respectively, which altered the tyrosine in CSSL28 to a termination codon in 9311 (Fig. S3). In addition, the promoter region was also altered at 17 sites between CSSL28 and 9311. Functional defects in the SD1 gene result in serious $\mathrm{PH}$ changes. 

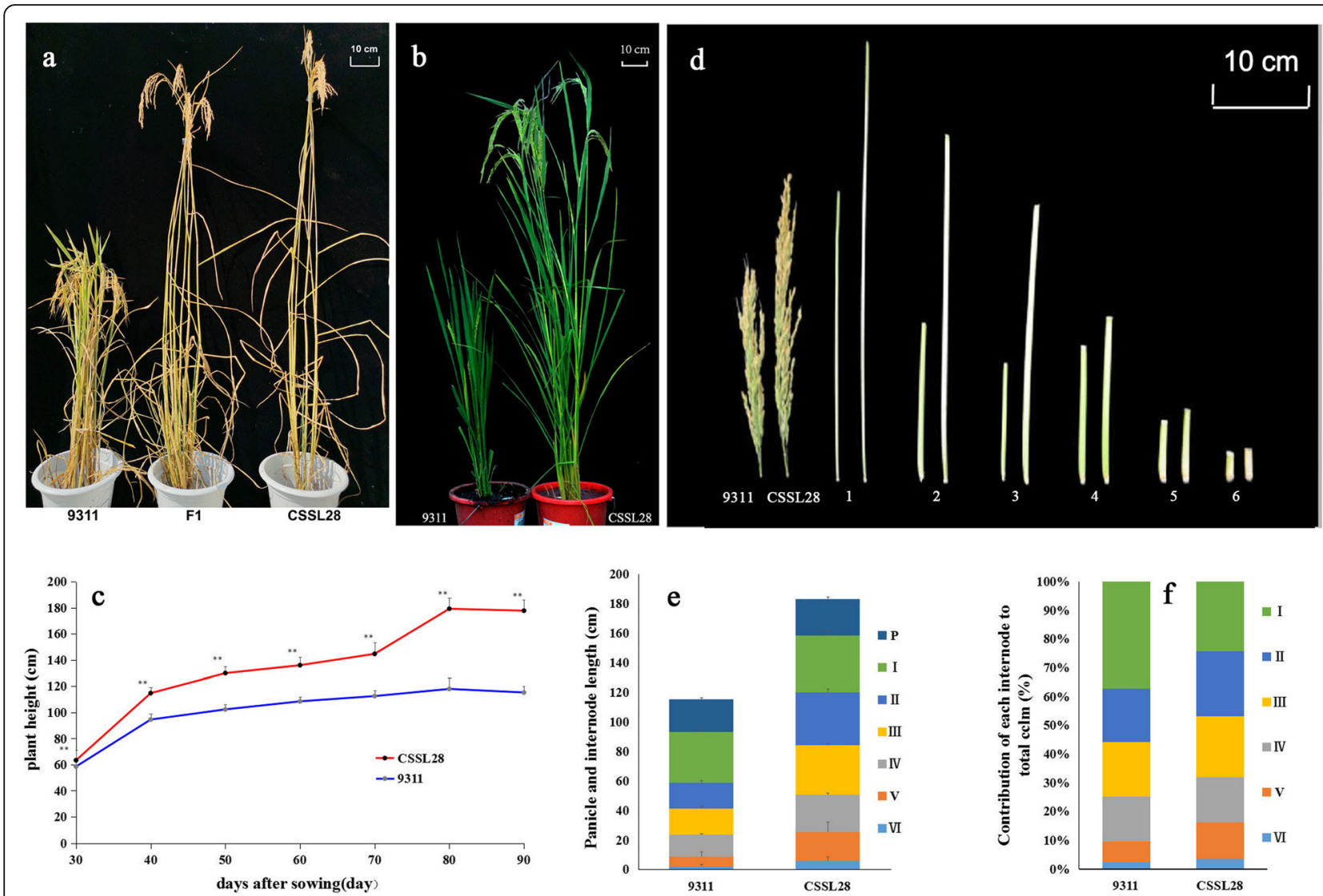

Fig. 1 Gross morphology of CSSL28 and 9311 rice lines. $\mathbf{a}$ and $\mathbf{b}$ Plant phenotypes of CSSL28, 9311 and individuals from the CSSL28/9311 $\mathrm{F}_{1}$ generation. c Dynamic comparison of plant heights between CSSL28 and 9311 at different growth stages; all data are provided as means \pm SDs $(n=20)$. $\mathbf{d}$ The appearances of the panicles and internodes of CSSL28 and 9311; 1-6 indicate internodes from head to base. e Comparison of the lengths of the panicles and internodes between CSSL28 and 9311; data are averages of the lengths of the panicles and internodes of the main culms $(n=50)$. $\mathbf{f}$ Schematic representation of internode elongation patterns of CSSL28 and 9311. **significant at $P<0.01$

Therefore, we hypothesized that $q C L 1.2$ is the $S D 1$ gene and that the extremely high $\mathrm{PH}$ of CSSL28 results from thewild riceSD1 allele.

\section{Gene expression analysis}

To investigate the expression patterns and regulatory network of the novel allele of the $S D 1$ gene, total RNA from seedlings of CSSL28 and 9311 at 5, 15 and $30 \mathrm{~d}$ after germination were isolated for a real-time PCR analysis. SD1 and genes involved in GA synthesis (EUI1) and GA signaling (SLR1 and GID1) were selected (Fig. 4). For $S D 1$, the expression level was high at $5 \mathrm{~d}$ after germination in both CSSL28 and 9311, and the expression level in 9311 was higher than that in CSSL28. At 15 and $30 \mathrm{~d}$ into the seedling stage, the expression level of $S D 1$ decreased in both CSSL28 and 9311.

For theSLR1 gene, which encodes a DELLA protein, and the GA receptorgene GID1, the expression levels were low at $5 \mathrm{~d}$ after germination and significantly increased at the 15th day of the seedling stage. The expression levels of the two genes in 9311 were much higher than those in
CSSL28 and then significantly decreased by the 30th day of the seedling stage. The same expression patterns were also found for the EUI1 gene, which correlates with the internode lengths at the top of rice stems.

\section{Nucleotide diversity and haplotype network analyses of the sd1 gene}

Using the rice functional genomics-based breeding database (http://www.rmbreeding.cn/index), the qCL1.2 (SD1) gene coding and promoter region sequences from 2822 rice varieties were aligned. Haplotype and genetic diversity analyses were carried out using the data of 2822 cultivated rice PH phenotypes. Abundant genetic variations were detected at the $L O C_{-}$Os01g66100 site in the 2822 cultivated rice accessions (Fig. 5). The SD1 coding region contained 27 non-synonymous SNP/InDel sites. In total, 33 haplotypes with more than 5 individuals were selected, and a total of 20 variation sites were retained (Fig. 5a). As shown in Fig. 6, a network was constructed using the major haplotypes for the SD1 coding region. The 33 haplotypes were basically divided into 

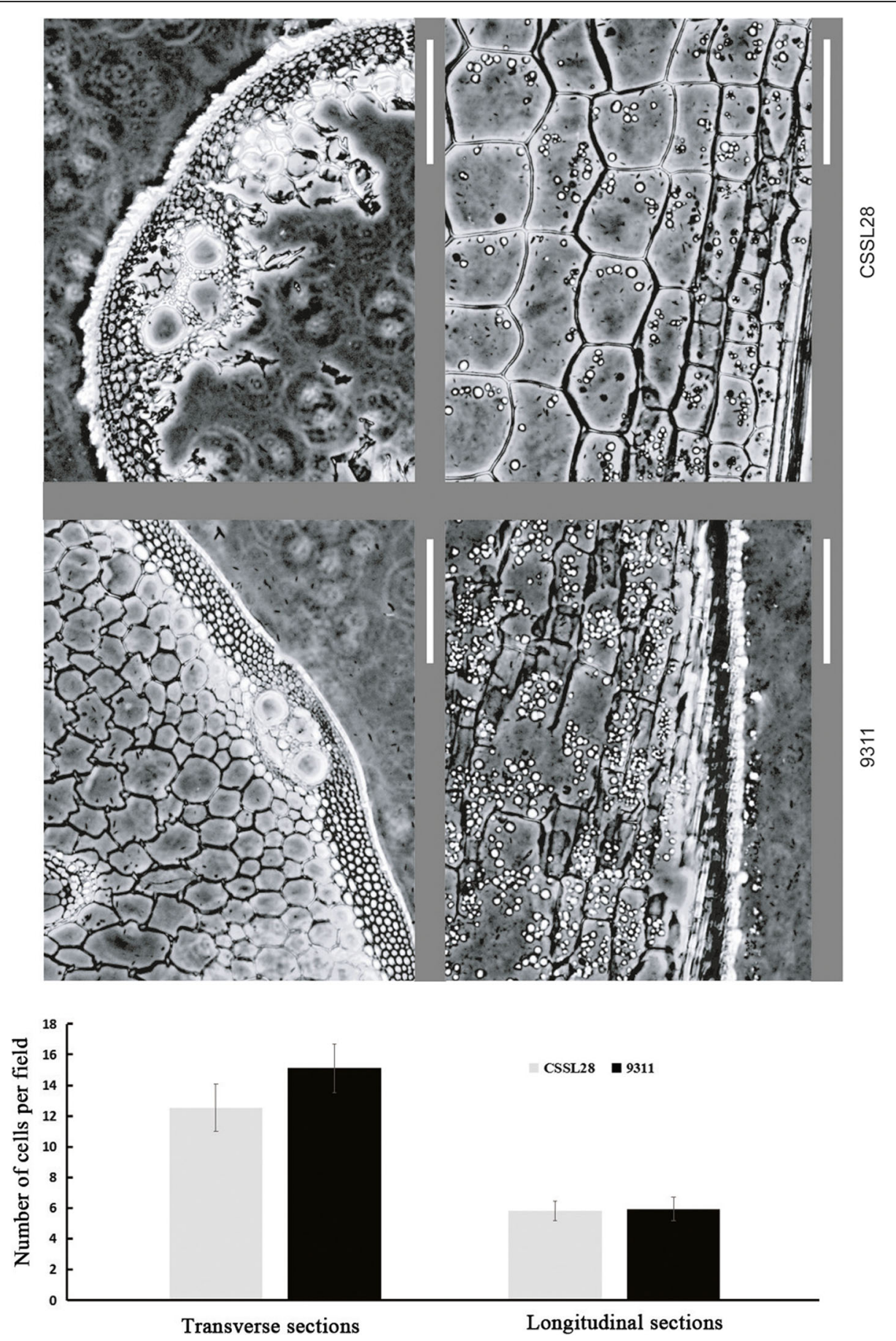

Fig. 2 Morphological characterization of the stems of CSSL28 and 9311 rice plants. Transverse and longitudinal sections of the third internode from the main culm at the heading stage. The statistical comparisons of the numbers of cells per field between CSSL28 and 9311 are shown below

three groups. The left group contained 8 haplotypes and $95.5 \%$ of the japonica rice samples, and the middle group contained 16 haplotypes and $89.1 \%$ of the indica rice samples (Fig. 6a). Associations between haplotypes and $\mathrm{PH}$ were also analyzed (Fig. 6b). Among the 24 haplotypes in the left and middle groups,97.6\% accessions having $\mathrm{PH}$ values greater than $130 \mathrm{~cm}$, and 95.5\% of the samples having $\mathrm{PH}$ values between 110 and $130 \mathrm{~cm}$ were in this group. In the right group, most of the $\mathrm{PH}$ values were less than $90 \mathrm{~cm}$, and 


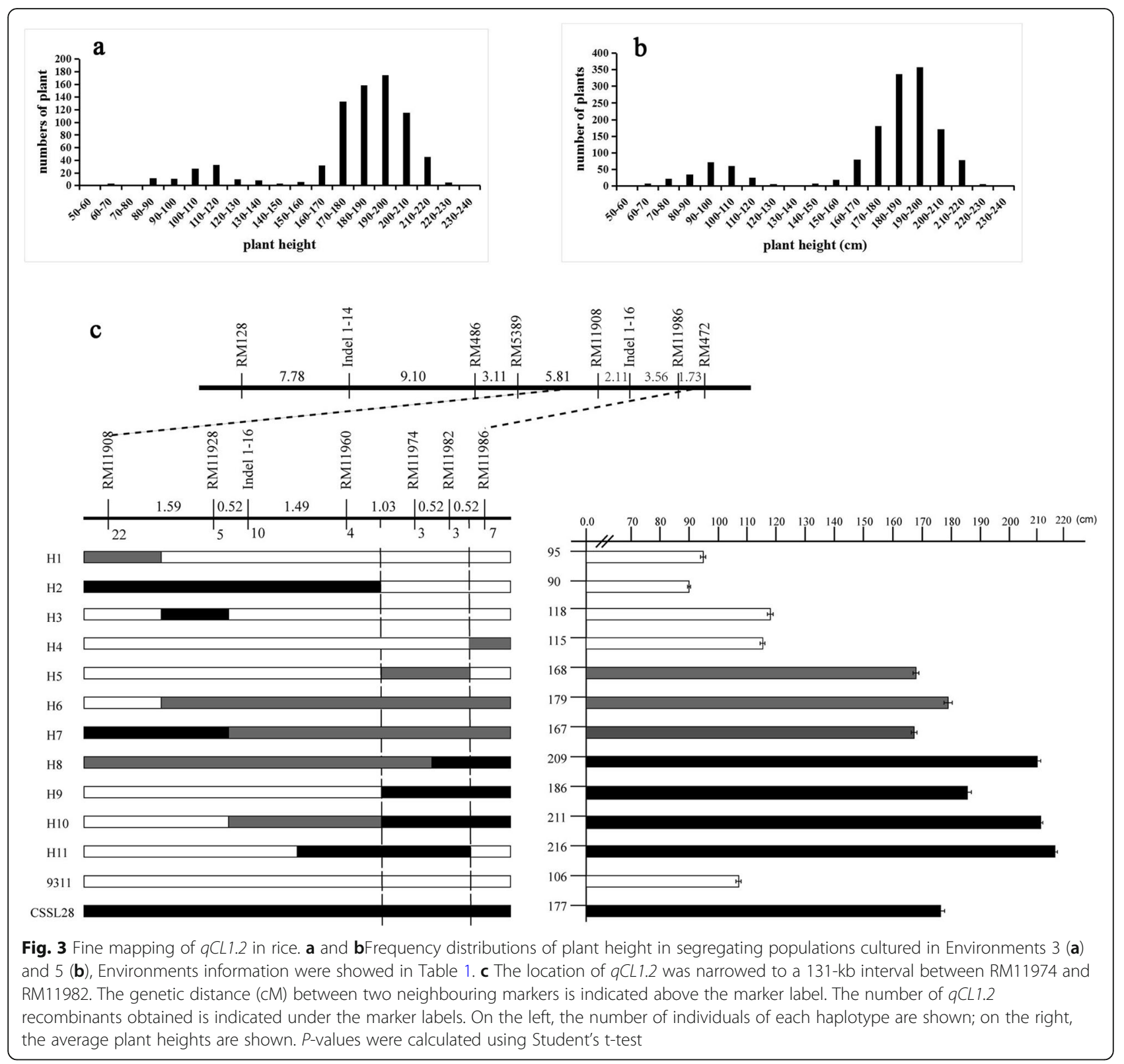

$50.69 \%$ of samples having $\mathrm{PH}$ values between 70 and $90 \mathrm{~cm}$ were in this group.

As shown in Fig. 5a, SNPs at nt 299 and 1019 in the SD1 coding region differentiated japonica and indica rice. The amino acids at the two sites were glutamate (E) and glutamine $(\mathrm{Q})$, respectively, in japonica, and glycine (G) and arginine (R), respectively, in indica. More than 98\% of the indica accessions carried the SD1-GR allele, as in $q$ CL1.2. Most of the japonica accessions carried the SD1-EQ allele. However, these two SNPs did not affect $\mathrm{PH}$. Compared with other haplotypes, the InDels in haplotypes H_13, H_14, H_21, H_22, H_23, H_24, H_29 and $\mathrm{H} \_33$ resulted in frame-shifts or translational termination, leading to the dwarf plant phenotype.
Additionally, in the H_20of 9311, the SNP at nt 1026 led to dwarfed plants.

A network for the $S D 1$ promoter sequence was also constructed using the same database. The $S D 1$ promoter region contained $51 \mathrm{SNP} / \mathrm{InDel}$ sites, and a total of 31 haplotypes having more than 10 individuals were selected (Fig. 5b). As shown in Fig. 7a, three haplotypes, H_3, 7 and 28 , contained $90.78 \%$ of the japonica individuals, while $93.88 \%$ of the indica individuals were in the other haplotypes. This finding suggested that SNPs at nt $35,93,242,412,447,537,853,1164$ and 1247 of the $s d 1$ promoter region (Fig. 5b) clearly differentiated between japonica and indica. Most accessions in H_21, 24, 29, 23 and 27 are 'others,' suggesting that SNPs at nt 35, 734, 

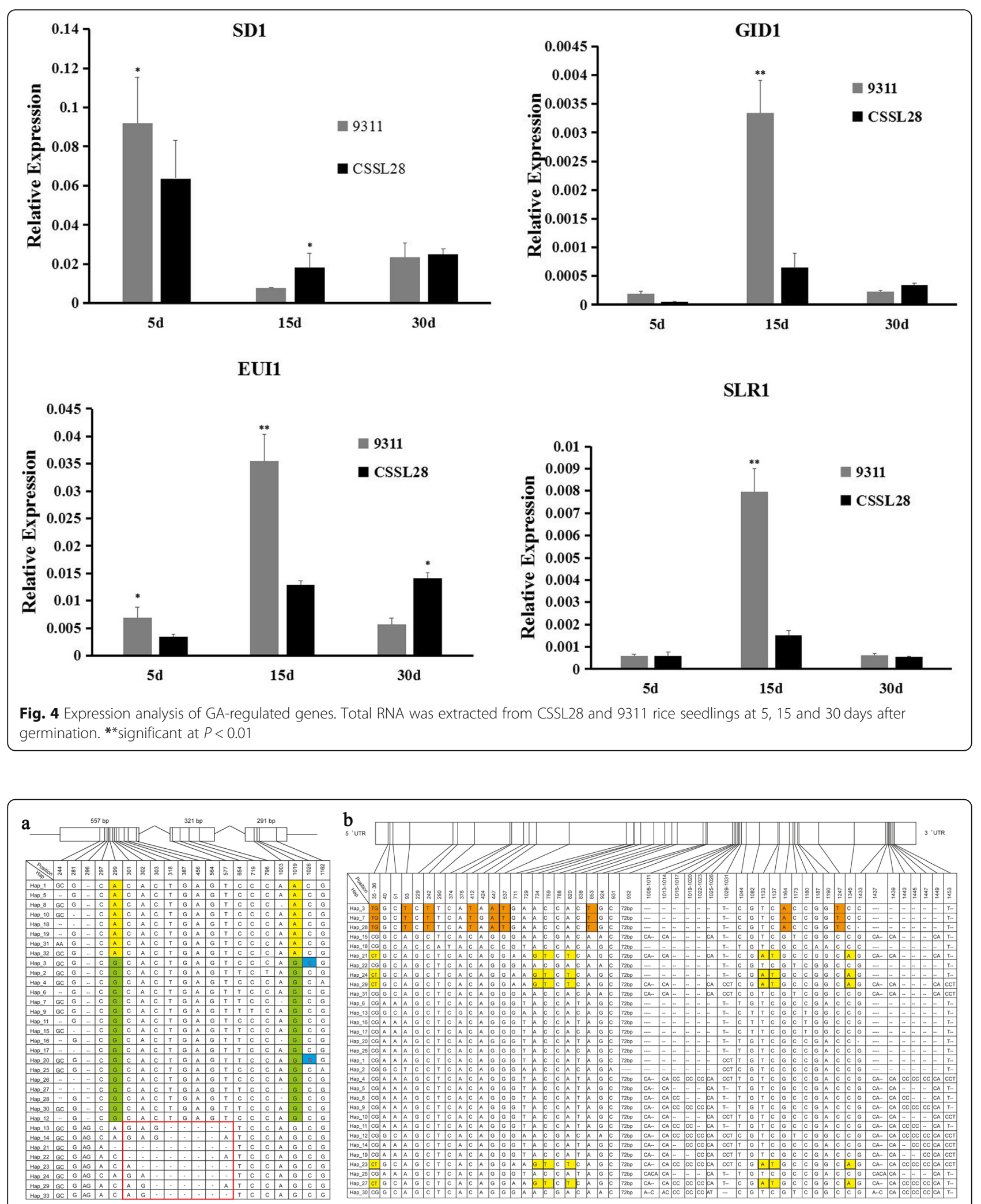

Fig. 5 Haplotype analysis of the SD1 gene in rice. a Major haplotypes (haplotypes carried by more than five accessions) of the SD1 coding region in the whole population based on non-synonymous SNPs data. Different colors at nucleotides 299 and 1019 represent japonica and indica. H_3 contains qCL1.2, the SNPs in blue and red rectangles represent FNPs for plant height. b Major haplotypes (haplotypes carried by more than 10 accessions) of the SD1 promoter region. SNPs in orange differentiate between japonica and indica, SNPs in yellow are specific to other accessions 


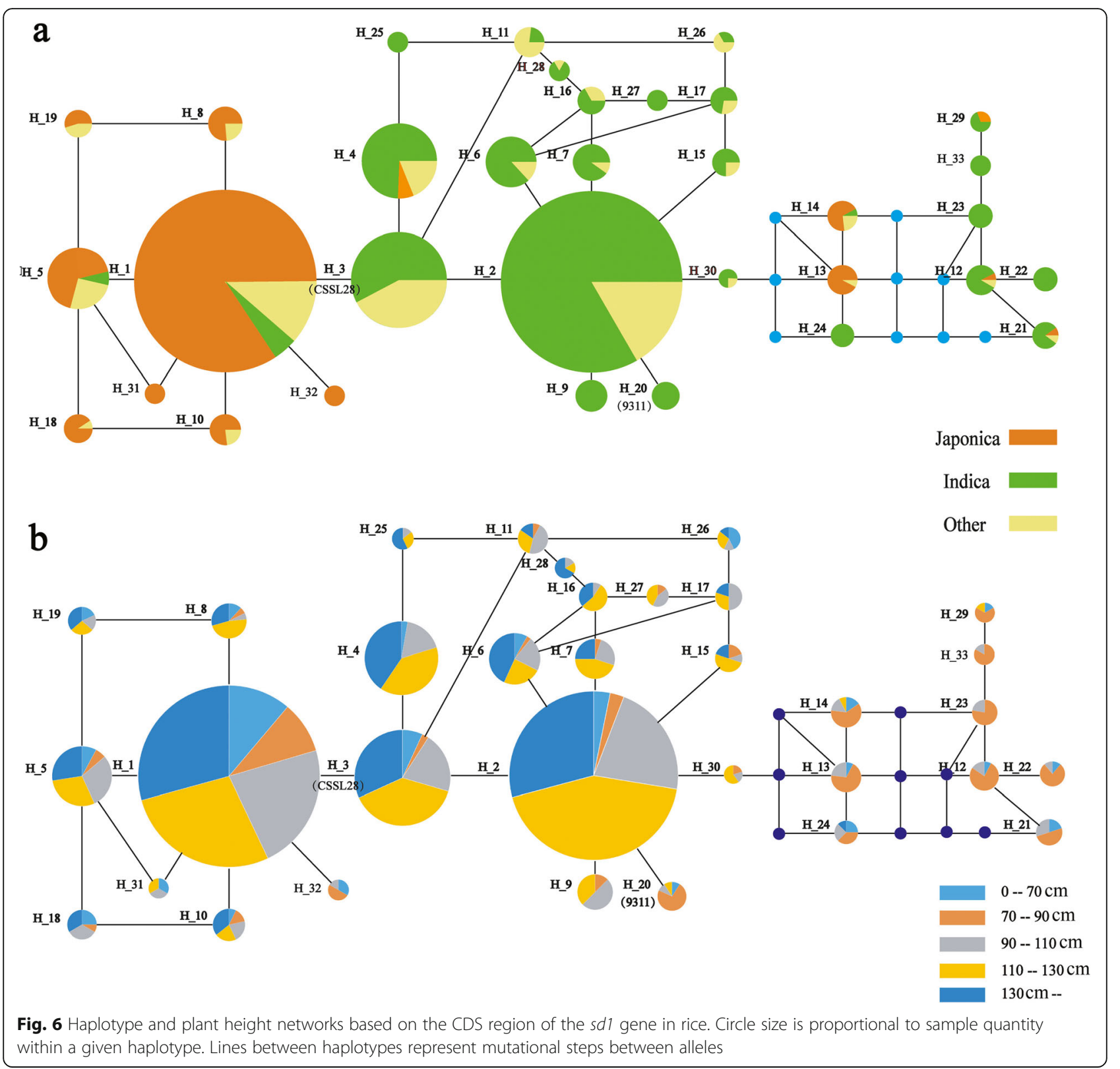

759, 820, 1133, 1137 and 1345 differentiated between indica and others. Furthermore, no SNP or haplotype was found associated with $\mathrm{PH}$ in Fig. 7b, indicating that the $\mathrm{PH}$ of rice is mostly controlled by the SD1 protein function but not the gene expression level.

\section{Discussion}

Wild rice is a crucial germplasm resource not only for cultivated rice breeding but also rice domestication studies. $\mathrm{PH}$ is a complex trait controlled by multiple genes. Although numerous dwarf mutants in rice have been described during the past decades, the exact functions of genes from wild relatives remains unclear $[4,8]$. In this study, we described a novel allele of a classic rice dwarf mutant, $s d 1$, which was first described as a "green revolution" gene in the 1960's [8, 20]. CSSLs are an excellent platform for studying wild alleles in cultivated rice genetic backgrounds. In our laboratory, a set of CSSLs of wild rice was constructed and used for gene discovery, resulting in the discovery of many QTLs related to important agronomic traits $[10,17]$. In the current study, a main PH QTL, $q C L 1.2$, was identified. $q C L 1.2$ was detected in four environments. A NIL of qCL1.2,CSSL28, had a greater $\mathrm{PH}$ than the recurrent parent (Fig. 1), and the $F_{2}$ population demonstrated a perfect 3:1 segregation ratio. These data showed that $q C L 1.2$ was a dominant locus that can significantly promote $\mathrm{PH}$. Using the genetic segregation populations, the location of 


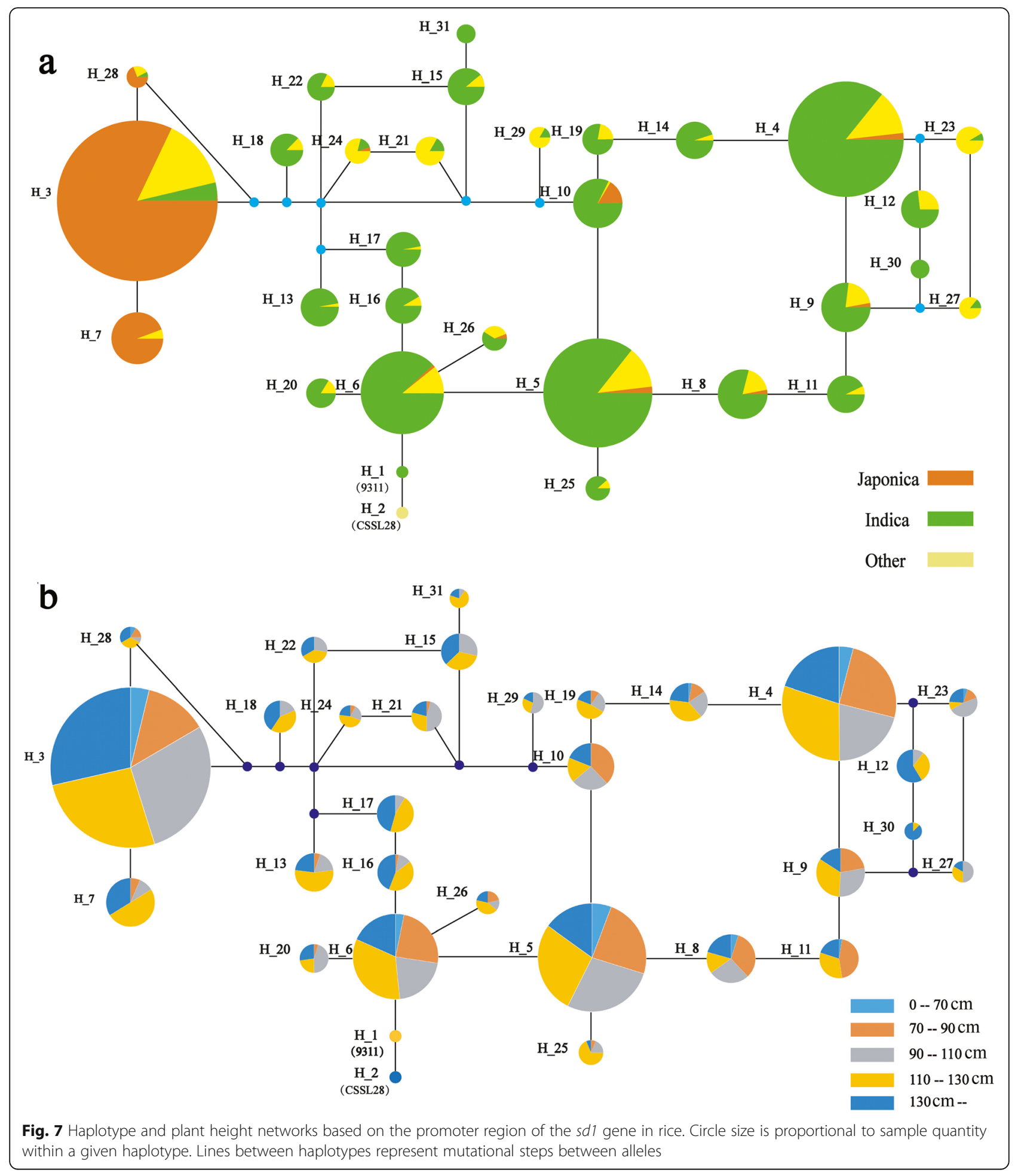

$q C L 1.2$ was narrowed to a 131-kb interval. The "green revolution" gene sd1 (LOC_Os01g66100) was identified in this region. One SNP found in the third exon led to translational termination. We also designed special primers for LOC_Os01g66100 detection in the CSSL28/9311 $\mathrm{F}_{2}$ population. All the individuals harboring the wild rice allele showed greater $\mathrm{PH}$ values than individuals harboring the 9311 allele (data not shown). This observation confirmed that $q C L 1.2$ was the wild allele of the SD1 gene.

The rice genome carries at least two GA20ox genes (GA20ox-1 and GA20ox-2). SD1 corresponds to 
GA20ox-2 and plays essential roles in GA biosynthesis and signal transduction processes $[8,21]$. There were many nucleotide changes in the promoter sequences of qCL1.2 (SD1) between the two parents (Fig. S3). Although the transcript levels of $S D 1$ differed between CSSL28 and 9311, the expression patterns in the two parents were similar (Fig. 4), indicating that the CSSL28 phenotypic changes were mainly caused by changes in the SD1 protein's function. Three genes involved in GA signaling, EUI1, SLR1 and GID1,were expressed at significantly higher levels in 9311 than CSSL28 at $15 \mathrm{~d}$ after germination. GID1 encodes a soluble receptor for GA [20], and SLR1 is a rice DELLA protein that binds to GID1 [22, 23]. Both GID1 and SLR1 undergo negative feedback regulation by GA signaling, as well as $S D 1$ andGA20ox genes. We deduced that the high $\mathrm{PH}$ of CSSL28 was induced by active SD1, and feedback regulated by the GID1-SLR1 pathway through GA signaling. EUI1, encoding a putative cytochrome P450 monooxygenase, regulates internode elongation by modulating GA responses in rice. Overexpression of EUI1 gave rise to the GA-deficient-like phenotypes [24]. CSSL28 had elongated internodes compared with 9311(Fig. 1), which might be regulated by active SD1 through repressedEUI1. A transgenic experiment should performed to confirm the exactly function of this wild allele.

Plant architecture was an essential target of artificial selection during both rice domestication and is still a target of modern breeding. In this study, two SNPs of SD1, nt 299 in the first exon (A/G, E to G) and 1019 in the third exon (A/G, Q to R), clearly differentiatedjaponica and indica. These two SNPs were firstreported by Asano et al. (2011) as key natural variations involved in rice domestication [19]. The results here were consistent with those of Asano et al. (2011). However, these two SNPs were not associated with PH. Most japonica accessions carried SD1-EQ (in the right group of Fig. 6) and had high $\mathrm{PH}$ values. Almost all the indica accession in the left group of Fig. 6 had short PH values. In the right group of Fig.6, the eight haplotypes of mostly short individuals indicated that eight InDels, 1-bp deletions at nt $301,302,303,318,387,456,564$ and 577 , had been specifically selected during indica domestication (Fig. 5a, red rectangle). In $\mathrm{H} \_13$ and 14, most of the samples were japonica and carried A at nt 299 and G at nt 1019; this is an intermediate allele SD1-ER, which was not identified in Asano et al.'s paper (2011). This discrepancy may be due to that fact that they used only 72 rice accessions, while we used more than 2800 landraces, including 854 japonica and 1789 indica. For H_20, most of the accessions were short, although it appeared in the middle group of Fig. 6, because the FNP at nt 1026 led to the translational termination of SD1.
In the promoter region, only 3 haplotypes were represented in japonica, while there were 28 haplotypes in indica rice. This finding was consistent with Asano et al. (2011) who determined that the nucleotide diversity of the SD1 flanking region in japonica was much lower than in indica. However, no SNP was associated with $\mathrm{PH}$, indicating that artificial selection only occurred for the SD1 coding region during the differentiation of $j a-$ ponica and indica. Additionally, it is the distinct $S D 1$ alleles, not their expression levels, that played an active role in $\mathrm{PH}$ during rice domestication. Our results revealed a new allele of the "green revolution" gene $S D 1$ from wild rice, which increased the PH in a NIL. Eight InDels and one FNP in the SD1 coding region were selected during rice domestication, in parallel with japonica and indica differentiation. Our study provides new insights into the functions and evolution of this gene.

\section{Conclusions}

In this study, a novel allele of SD1 gene was identified from wild rice using a set of CSSLs. The wild allele of $S D 1$ can significantly promote rice internode length by regulating the expression levels of genes involved in gibberellin biosynthesis and signal transduction. Two key FNPs as key natural variations involved in rice domestication were previously reported, our findings provide new evidence for artificial $\mathrm{PH}$ selection during rice domestication and differentiation. The novel SD1 allele and the new FNPs found in this study provide an increased understanding of rice $\mathrm{PH}$-targeted breeding.

\section{Methods}

\section{Plant material and field trial}

A set of 198 CSSLs produced from common wild rice (O. rufipogon) as the donor and an elite indica variety, 9311, as the recurrent parent was developed in our laboratory as previously reported [10]. The CSSLs and 9311 were grown under five environmental conditions as shown in Table 1. Each plot consisted of rows having 10 plants. In total, 40 plants of each genotype in each plot were planted with a $10 \times 27-\mathrm{cm}$ spacing. Crop management and disease and pest control were carried out in accordance with local recommendations.

\section{Phenotypic survey and histological observations}

The $\mathrm{PH}$ was measured from the ground surface to the tallest panicle. Internodes from top to bottom were named $P$ (panicle), first through sixth. The internodes of each stem at the mature stage were fixed in a FAA solution, containing $50 \%$ ethanol, $5 \%$ acetic glacial and 3.7\% formaldehyde, for $24 \mathrm{~h}$ at $4{ }^{\circ} \mathrm{C}$ and were then dehydrated in a graded ethanol series (70, 80, 90 and 100\% twice). The microscopic images were captured by a Leica Digital Camera system. Stem cuticles were prepared for light 
microscopic observations according to standard preparation techniques [25].

\section{Gene expression analysis}

The expression materials CSSL28 and 9311 were planted in an artificial climate chamber (model: XT5408CC320TL2H, Xutemp Tech Compay, Hangzhou, China), and the humidity was stably controlled at $80 \% \pm 5 \%$.An 8-h light/12-h dark photocycle was used, and the temperature was controlled at $\sim 28^{\circ} \mathrm{C}$.After $5 \mathrm{~d}$ of hydroponic culturing in a light incubator, the culturing was continued in a mixed nutrient soil, to ensure uniform growth conditions. Sampling was carried out at 5, 15 and $30 \mathrm{~d}$ of culturing. Liquid nitrogen was immediately injected to prevent RNA degradationafter sampling. RNA was extracted using TRIzol reagent (Invitrogen, CA, USA) and treated with DNase I (Invitrogen).cDNA was synthesized using SuperScript III Reverse Transcriptase (Invitrogen). A quantitative analysis of gene expression was performed on an Applied Biosystems 7500 Real-Time PCR System using SYBR Premix Ex Taq (TaKaRa, Otsu, Japan). Data were analyzed using a relative quantitative method [26]. Each real-time PCR reaction had three duplications, and the Actin gene of rice was used as an internal reference.

\section{DNA extraction, PCR protocol and molecular marker analysis}

DNA was extracted from rice seedling individuals as previously described [27]. The PCR reaction volume was $15 \mu \mathrm{L}$, containing $1.2 \mu \mathrm{L}$ of template DNA, $0.075 \mu \mathrm{L}$ of Taq DNA polymerase, $1.5 \mu \mathrm{L}$ of $10 \times$ buffer, $0.3 \mu \mathrm{L}$ of 10 $\mathrm{mM} \mathrm{dNTP}, 0.6 \mu \mathrm{L}$ of $10 \mu \mathrm{mol} / \mathrm{L}$ forward and reverse primers and $10.725 \mu \mathrm{LddH}_{2} \mathrm{O}$.After $5 \mathrm{~min}$ of predenaturation at $95^{\circ} \mathrm{C}, 33$ cycles of $94^{\circ} \mathrm{C}$ for $30 \mathrm{~s}, 56^{\circ} \mathrm{C}$ for $30 \mathrm{~s}$ and $72{ }^{\circ} \mathrm{C}$ for $30 \mathrm{~s}$ were performed, followed by $72^{\circ} \mathrm{C}$ for $7 \mathrm{~min}$. The PCR products were electrophoresed on a $4 \%$ polyacrylamide gels and visualized by silver staining.

The SSR primers used in this study werepreviously published [28, 29], InDel primers were designed in our laboratory [16]. The other primers used in the experiment were based on the 9311 reference genome sequence and were designed online at the NCBI website (Https://www.ncbi.nlm.nih.gov/). Alignments were performed on the Grammer website to ensure the accuracy of the location and the specificity of the primers. Sequences of all molecular markers used in this study are shown in Table S2.

\section{QTL analysis and candidate gene prediction}

The analytic softwareQTL IciMapping [30] was used to processes genotypic and phenotypic data for the CSSL population and the offspring. The method used in this studywas a complete interval-additive model, and the
LOD threshold was defined as 2.5. Thus, a LOD value greater than or equal to 2.5 indicated that there is a valid QTL at the site. Naming was performed in accordance with the McCouch method [31].

\section{Network and genetic diversity analyses}

Data on the SD1 gene sequences of 2822 rice accessions were compiled from the Rice Functional Genomics and Breeding Database (http://www.rmbreeding.cn/snp3k) [32]. This sub-database is a global resource that contains tools, such as a polymorphism information retrieval function, genome browser visualization system, and data export system, for specific genomic regions. All the SNPs located in the promoter and coding regions of the SD1 gene were extracted based on the genome gff3 annotation. The haplotype analysis was performed using Perl scripts, and only non-synonymous SNPs were considered. Numbers of haplotypes and haplotype diversity levels were determined using DnaSPv5 software (http:// www.ub.edu/dnasp) and introduced into the NETWORK 5.0program for haplotype network construction [33].

\section{Supplementary information}

Supplementary information accompanies this paper at https://doi.org/10. 1186/s12863-020-00868-w.

Additional file 1 Fig. S1. The distribution of PH of CSSLs under five environments (E1-5).

Additional file $\mathbf{2}$ Fig. S2. a, linkage map of SSR/InDel markers used in CSSLs genotyping, the introgrissive segements of CSSL28 were marked as red; b, photos of CSSL28 (right) and 9311 (lift) seedlings at 5, 15, 30 days after germination (DAG)

Additional file $\mathbf{3}$ Table S1. Gene prediction analysis in delimitation region of $\mathrm{QCL} 1.2$

Additional file 4 Fig. S3. Comparison of SD1 between rice lines 9311 and CSSL28.a SNPs found in the SD1 promoter and coding regions. b Sequence alignment of the SD1 gene

Additional file 5 Table S2. Infomations of SSR and InDel markers used in this study.

\section{Abbreviations}

PH: Plant height; CSSL: Chromosome segment substitution line;

QTL: Quantitative trait locus; SSR: Simple sequence repeats; InDel: Insert/ deletion; SNP: Single nucleotide polymorphism; FNP: Functional nucleotide polymorphism; PCR: Polymerase chain reaction

\section{Acknowledgements}

We thank Dr. Tianqing Zheng and Dr. Wensheng Wang in Institute of Crop Science, Chinese Academy of Agricultural Sciences, for their assistance in haplotype network analyses.

\section{Authors' contributions}

LZ, JHand YWcontributed equally to this work. LZ and WQ performed the experiments and wrote the manuscript. YWcontributed to

manuscriptrevising. JH, YW, RX, and ZYall contributed to PCR genotyping, ZZ, $S L, Y T, X Z$ and FL contributed to field experiment. JW, YS, JL, YC, LFZ and YC analysed the phenotypic data. JL, WQ and QY designed the experiment. All authors read and approved the final manuscript. 


\section{Funding}

This study was supported by a grant from the National Natural Science Foundation of China (No. 31471471), the National Key Research and Development Program of China (2016YFD0100101) and the Agricultural Science and Technology Innovation Program of Chinese Academy of Agricultural Science to Weihua Qiao. The funders had no role in the study design, data collection, and analysis, decision to publish or preparation of the manuscript.

\section{Availability of data and materials}

The datasets used and/or analysed during the current study available from the corresponding author on reasonable request.

\section{Ethics approval and consent to participate}

The authors declare that this study complies with the current laws of the countries in which the experiments were performed.

\section{Consent for publication}

Not applicable.

\section{Competing interests}

The authors declare that they have no competing interests.

\section{Author details}

${ }^{1}$ Qingdao Agricultural University, Qingdao 266109, China. ${ }^{2}$ Institute of Crop Science, Chinese Academy of Agricultural Sciences, Beijing 100081, China.

${ }^{3}$ Shanxi Agricultural University, Shanxi province, Taigu, China. ${ }^{4}$ Nanjing Agricultural University, Nanjing 571100, China.

\section{Received: 11 February 2020 Accepted: 28 May 2020}

\section{Published online: 11 June 2020}

\section{References}

1. Fageria NK. Yield physiology of rice. J Plant Nutr. 2007:30:843-79.

2. Khush GS. What it will take to Feed 5.0 Billion Rice consumers in 2030. Plant Mol Biol. 2005:59:1-6.

3. Wang YH, Li JY. Molecular basis of plant architecture. Annu Rev Plant Biol. 2008:59:253-79.

4. Hong Z, Ueguchi-Tanaka M, Umemura K, Uozu S, Fujioka S, Takatsuto S, et al. A rice brassinosteroid-deficient mutant, ebisu dwarf ( $d 2$ ), is caused by a loss of function of a new member of cytochrome P450. Plant Cell. 2003; 15:2900-10.

5. Spielmeyer W, Ellis MH, Chandler PM. Semidwarf (sd-1), "green revolution" rice, contains a defective gibberellin 20-oxidase gene. Proc Natl Acad Sci U S A. 2002:99:9043-8

6. Mann S, Ozin GA. Synthesis of inorganic materials with complex form. Nature. 1996:382:313-8.

7. Khush GS. Green revolution: preparing for the 21st century. Genome. 1999 42:646-55.

8. Sasaki A, Ashikari M, Ueguchi-Tanaka M, Itoh H, Nishimura A, Swapan D, et al. Green revolution: a mutant gibberellin-synthesis gene in rice - new insight into the rice variant that helped to avert famine over thirty years ago. Nature. 2002;416:701-2.

9. Monna L, Kitazawa N, Yoshino R, Suzuki J, Masuda H, Maehara Y, et al. Positional cloning of rice semidwarfing gene, $s d-1$ : Rice "green revolution gene" encodes a mutant enzyme involved in gibberellin synthesis. DNA Res. 2002;9:11-7.

10. Qiao WH, Qi L, Cheng ZJ, Su L, Li J, Sun Y, et al. Development and characterization of chromosome segment substitution lines derived from Oryza rufipogon in the genetic background of 0 . sativa spp. indica cultivar 9311. BMC Genomics. 2016;17:580.

11. Wang ZY, Second G, Tanksley SD. Polymorphism and phylogenetic relationships among species in the genusOryzaas determined by analysis of nuclear RFLPs. Theor Appl Genet. 1992;83:565-81.

12. Sun CQ, Wang XK, Li ZC, Yoshimura A, Iwata N. Comparison of the genetic diversity of common wild rice (Oryza rufipogon Griff.) and cultivated rice $(O$. sativa L.) using RFLP markers. Theor Appl Genet. 2001;102:157-62.

13. Huang $X H$, Wei $X H$, Sang $T$, Zhao QA, Feng Q, Zhao Y, et al. Genome-wide association studies of 14 agronomic traits in rice landraces. Nat Genet. 2010; 42:961-7.
14. Li CB, Zhou AL, Sang T. Rice domestication by reducing shattering. Science. 2006:311:1936-9.

15. Ali ML, Sanchez PL, Yu SB, Lorieux M, Eizenga GC. Chromosome Segment Substitution Lines: A Powerful Tool for the Introgression of Valuable Genes from Oryza Wild Species into Cultivated Rice (O. sativa). Rice. 2010;3:218-34.

16. Qi L, Sun Y, Li J, Su L, Zheng XM, Wang XN, et al. Identify QTLs for grain size and weight in common wild rice using chromosome segment substitution lines across six environments. Breed Sci. 2017;67:472-82.

17. Qi L, Ding YB, Zheng XM, Xu R, Zhang LZ, Wang YY, et al. Fine mapping and identification of a novel locus qGL12.2 controls grain length in wild rice (Oryza rufipogon Griff.). Theor Appl Genet. 2018;131:1497-508.

18. Li J, Xu R, Wang CC, Qi L, Zheng XM, Wang WS, Ding YB, et al. A heading date QTL, GHD7.2, from wild rice (Oryza rufipogon) delays flowering and shortens panicle length under long-day conditions. Sci Rep. 2018;8:2928.

19. Asano K, Yamasaki M, Takuno S, Miura K, Katagiri S, Ito T, et al. Artificial selection for a green revolution gene during japonica rice domestication. Proc Natl Acad Sci U S A. 2011;108:11034-9.

20. Ueguchi-Tanaka M, Ashikari M, Nakajima M, Itoh H, Katoh E, Kobayashi M, et al. GIBBERELLIN INSENSITIVE DWARF1 encodes a soluble receptor for gibberellin. Nature. 2005;437:693-8.

21. Yang PD, Zhao DY, Margolese DI, Chmelka BF, Stucky GD. Generalized syntheses of large-pore mesoporous metal oxides with semicrystalline frameworks. Nature. 1998;396:152-5.

22. Ikeda A, Ueguchi-Tanaka M, Sonoda Y, Kitano H, Koshioka M, Futsuhara Y, et al. Slender rice, a constitutive gibberellin response mutant, is caused by a null mutation of the SLR1 gene, an ortholog of the height-regulating gene GAI/RGA/RHT/D8. Plant Cell. 2001;13:999-1010

23. Fukazawa J, Mori M, Watanabe S, Miyamoto C, Ito T, Takahashi Y. DELLAGAF1 complex is a Main component in gibberellin feedback regulation of GA20 oxidase 2. Plant Physiol. 2017;175:1395-406.

24. Luo A, Qian Q, Yin H, Liu X, Yin C, et al. EUl1, encoding a putative cytochrome $\mathrm{P} 450$ monooxygenase, regulates internode elongation by modulating gibberellin responses in rice. Plant Cell Physiol. 2006;47:181-91.

25. Leng Q, Yang H, Yang Q, Zhou JP. Variation of cuticle micromorphology of Metasequoia glyptostroboides (Taxodiaceae). Bot J Linn Soc. 2001;136:207-19.

26. Livak KJ, Schmittgen TD. Analysis of relative gene expression data using real-time quantitative PCR and the $2^{-}$CT method. Methods. 2001:25:402-8.

27. RamkumarG SA, Pandey M, Sakthivel K, Shobha Rani N, Sudarshan I, et al. Development of a PCR-based SNP marker system for effective selection of kernel length and kernel elongation in rice. Mol Breed. 2010;26:735-40.

28. Cho YG, Ishii T, Temnykh S, Chen X, Lipovich L, McCouch SR, et al. Diversity of microsatellites derived from genomic libraries and GenBank sequences in rice (Oryza sativa L.). Theor Appl Genet. 2000;100:713-22.

29. Islam MR, Singh RK, Salam MA, Hassan L, Gregorio GB. Molecular diversity of stress tolerant Rice genotypes using SSR markers. Sabrao J Breed Genet. 2008:40:127-39.

30. Meng L, Li HH, Zhang LK, Wang J. QTL IciMapping: integrated software for genetic linkage map construction and quantitative trait locus mapping in biparental populations. Crop J. 2015;3:269-83.

31. Philn M, Tim P. Guidelines for common bean QTL nomenclature. Annu Rep. 2010:53:202-4.

32. Zheng $T Q$, Yu H, Zhang HL, Wu ZC, Wang WS, Tai SS, et al. Rice functional genomics and breeding database (RFGB): 3K-rice SNP and InDel subdatabase. Chin Sci Bull. 2015;60:367-71.

33. Librado P, Rozas J. DnaSP v5: a software for comprehensive analysis of DNA polymorphism data. Bioinformatics. 2009;25:1451-2.

\section{Publisher's Note}

Springer Nature remains neutral with regard to jurisdictional claims in published maps and institutional affiliations. 